\title{
The whole is greater than the sum of the parts
}

\author{
Louis J. Ptáček and Ying-Hui Fu
}

Department of Neurology, Institute for Human Cenetics, Weill Institute for Neuroscience, Kavli Institute for Fundamental Neuroscience, University of California San Francisco, San Francisco, California, USA.

0 ver the last 30 years, biomedical research has become increasingly interdisciplinary, and collaboration has become ever more important. Most investigators in biomedical research engage in collaboration. In this Viewpoint, we address a closer, long-term, and more committed collaboration in which two investigators coauthor a majority of their publications. Despite being independent investigators, each with an individual laboratory, the model that has evolved for us was to build an operation wherein the two labs function much like a single lab with two principal investigators. There are many examples of successful partnerships with overlapping research interests and complementary skills. Notable examples include John and Christine Seidman at Harvard University, Brown and Goldstein at the University of Texas Southwestern, and Lily and Yuh-Nung Jan at the University of California in San Francisco. However, this model is not common.

\section{Motivations for a scientific partnership}

A confluence of factors led to our collaboration. One of us (Louis Ptáček) was a young assistant professor and had an academic lab with rapid growth in research directions focused on human neurogenetics. It had become clear that to continue to be successful, it would be necessary to focus more and to jettison some projects to continue to make rapid progress on others. It was serendipitous that at that time; one of us (YingHui $\mathrm{Fu}$ ) had decided to return to academia after a productive five-year stint in the biotech industry. Since researchers don't take their work at a company with them to start an academic lab, she was considering opportunities for new areas on which to embark upon reentering academia. Thus, a partnership emerged such that a wider scope of interests could be pursued with shared leadership in an established research environment.
By this point, each of us had done quite a bit of work in Mendelian disease genetics, mapping and cloning human disease genes and mutations (1-5). These were gratifying and rewarding efforts that very directly led to better diagnosis of patients with those diseases. But we had begun to develop interests in deeper exploration of the function of proteins encoded by novel genes and characterization of functional consequences of disease-causing mutations. This was motivated by the hope for better therapies based on knowledge of pathophysiology. Moving into new areas always presents challenges, but is a logical consequence of discovery. In retrospect, our scientific partnership allowed risk taking that might otherwise have been too daunting. Critical to this transition was the shared interest in application of the emerging tools of modern human genetics to understanding pathophysiology of human neurological diseases. While some benefits of such a collaboration were obvious, some benefits and challenges were not. Looking back with the benefit of hindsight, we now recognize the serendipity of converging and complementary qualities that have contributed to ongoing success, but also recognize some things that could have been done differently.

\section{Our experience with scientific partnership}

The dawn of a new era in modern human genetics began with the proposition to map the human genome (6), which was foundational to the later sequencing of the human genome. Tools began to evolve for mapping and cloning human disease genes with identification of causative mutations. We were both fortunate to enter the field early during that exciting time. Not long thereafter, an opportunity to move into human behavioral genetics presented itself. We were both nervous about this, since behavioral genetics is so difficult, particularly in

Conflict of interest: The authors have declared that no conflict of interest exists.

Copyright: (c) 2021, American Society for Clinical Investigation.

Reference information: / Clin Invest. 2021;131(2):e145965. https://doi.org/10.1172/JCl145965.

humans. In fact, many thought this a "fool's errand," since behavioral genetics is so much easier in model systems. Serendipitously, our long-time collaborator Christopher Jones had recognized a family with a circadian trait of early morning awakening and early sleep times.

We've always known that there are "morning larks" and "night owls" in the population, but as we set out to characterize that first family, it became clear that this was not just a quantitative trait (7-9). There was striking Mendelian segregation of the trait in the original large Utah family, and together with Chris, we embarked on characterizing the phenotype and developing diagnostic criteria that were both sensitive and specific. This would be necessary to having any hope of mapping and cloning a causative gene. We called this trait familial advanced sleep phase (FASP).

Although much beautiful work in circadian genetics had begun with the work of Konopka and Benzer (10), the possibility of applying genetics to human sleep behavior was uncharted territory. As in many cases, this made the possibility even scarier, but also more exciting. As with all new frontiers, many challenges and obstacles presented themselves over the years. Our shared vision and conviction of the importance of understanding the molecular basis of human sleep reinforced this conviction and fortified our resolve for exploring this challenging new area.

Over the last two decades, this work has led to identification of a growing list of human circadian genes with mutations affecting circadian regulation with many novel insights into the molecular basis of regulation (8, 11-15). Even more exciting is the fact that a large majority of our FASP families don't have recognized mutations in any of the known candidate circadian genes. Our most recent whole exomesequencing efforts have identified three outstanding candidates as potential FASP genes that are in various stages of investigation (unpublished data). Thus, the established partnership has gone from describ- 
ing the first families with a Mendelian circadian trait to identification of genes and mutations leading to altered sleep schedule, which led to many novel insights into human circadian regulation. This represents a feat that would have been impossible for either of us to achieve alone.

More recently, we identified and reported the first pedigrees with a sleep homeostasis phenotype. Sleep experts have recognized the existence of natural short sleepers (NSS) in the population who require only four to six hours to feel well rested. But the report of a familial form of NSS (FNSS) was first published about a decade ago along with in vitro and in vivo functional characterization of causative gene/mutation (16). This work was also in close collaboration with Chris Jones. In the ensuing years, we've collected a large cohort of FNSS families and used whole exome sequencing to identify an additional five human sleep genes along with functional characterization in vitro and in vivo (refs. 17-20 and unpublished data). This is particularly exciting, since much less is known about regulation of human sleep requirements than about circadian regulation. Many more genes/mutations/molecular pathways for regulating human sleep are certain to come from studying our growing database of "unexplained" FNSS families. Again, it would be hard to imagine having moved into such new and exciting directions without the intellectual and moral support of partners in this undertaking.

Mutual respect is critical for any healthy collaboration. But for these types of partnerships to work well, it seems critical to be collaborating from a position of equality. Our partnership began with a significant amount of mutual respect growing from an overlap in research interests dating to our postdoctoral work on myotonic dystrophy and the nondystrophic myotonias. Hence, it was easy for us to feel completely equal as scientific partners in the collaborative work. At the same time, with one of us returning from industry into new areas of human genetic work, many did not view us as equal.

\section{Benefits of our scientific partnership}

A shared vision and passion for specific scientific questions provided powerful motivation. It was clear that in working together, there was a possibility of explor- ing a wider range of scientific questions than would have been possible for either of us alone. Our work has spanned from recognition and characterization of new clinical diseases and behavioral traits through deep in vitro biochemical and cell biological experiments toward understanding mechanism. This has required a broad spectrum of skills, even more so when we set out to extend this work to animal models with in vivo characterization of physiology and behavior. Having very invested partners with different training, strengths, and perspectives was critical here. Training programs (including medical scientist training programs) aim to give broad-based training and serve a very important place in biomedical research. At the same time, the bandwidth that any one person has for work in such diverse directions has limits and partnering with a trusted colleague increases the available capacity. In our experience, this is not simply additive. There have been tremendous synergies that have led to the whole being greater than the sum of the parts.

Such synergies can occur when each partner brings strengths to complement the other so that they together produce a much better outcome than would have been possible separately. We both recognized differences in our own understanding of clinical aspects versus technical aspects of cellular and molecular neuroscience techniques, our ability to import new technologies, our aptitude for facilitating outside collaborations, and administrative/organizational skills.

An essential component for fruitful and long-term collaborations is open communication. As in any relationship, differences of opinion can sometimes cause stress. But it is also an opportunity for exploring constructive compromises that are often more measured. It also helps to prevent one from getting too bogged down with his/her own strongly held opinions. Open discussions around differences of opinion may, in fact, be one of the primary benefits in close partnerships. Such discussions can affect all areas of the enterprise, from research direction, specific methodological approaches, analysis, and interpretation of data to administrative aspects of managing laboratories.

Another obvious benefit was the ability to split leadership responsibilities for dif- ferent research projects. It has always been helpful to have two separate perspectives for any project from individuals who are significantly invested. Differences of opinion are not a problem here - rather, they provide an opportunity to consider different options/strategies, much like the review process with which we are all familiar. They continually challenge us to be better.

\section{Challenges of long-term collaboration}

We view ourselves as completely equal partners, and we have colleagues locally, nationally, and internationally who share that view. However, we have also noticed systematic differences regarding how we are perceived by some. Peoples' perceptions of our individual contributions are often quite different from our own. On average, it seems that people are more willing to give credit to (a) a male versus a female; (b) a native English speaker versus someone who speaks English as a second (or third) language; (c) a career academic scientist versus one who has returned to academia from industry; and (d) a person with a gregarious and outgoing versus a more introverted personality. These are not universal biases, but are also not uncommon. We've recognized these types of biases at the level of interactions with individual scientists, at the departmental and institutional levels, and by the fields in which we have worked.

This collaboration has been a longterm learning process for us, and it is important to consider how we might better promote a culture of equality and appropriate recognition of individual accomplishments. What has worked best for us is to always keep the focus on the scientific questions and goals of discovery. This helps to focus attention on appropriately crediting the contributions of partners and the entire collaborative team. We also talk openly about collaboration, individual contributions of coauthors, and how to appropriately acknowledge collaborators' contributions with trainees in the hope of contributing to continued improvement in equality in the culture of science. It is also very important to mentor and encourage trainees in learning skills to help promote their own work appropriately. Ongoing attention needs to be paid to systemic discrimination of all types 


\section{Concluding remarks}

Our database of well-characterized families with FASP and FNSS has also begun to reveal connections between circadian clock/sleep and other interesting phenotypes such as mood, migraine, metabolism, and aging. It is our hope that this partnership (with a growing cohort of great collaborators) will continue to lead to discoveries related to human circadian regulation and sleep that will ultimately contribute to improved human health. Tremendous synergies are likely to result from such efforts.

Looking back after more than two decades of a successful scientific partnership, the benefits obviously exceed the obstacles. Nonetheless, we are mindful of the potential challenges and inequities that require some of our attention, energy, and actions.

\section{Acknowledgments}

We are grateful to a long list of students and postdoctoral fellows over the years who have done incredible work and contributed in significant ways to accomplishments of our labs and new directions in which they have developed that work. They have benefited by having co-mentors, but also have had to deal with having "two bosses," which is not always easy. Their accomplishments are significant in their own right. But these individuals also represent the next generation, leading their own labs, working in industry, teaching, and training future scientists. We are honored to have such great trainees and colleagues with a shared vision of discovery, with the ultimate goal of improving human health. Christopher Jones has been a wonderful collaborator on all of our work in circadian and sleep genetics. We are honored to have had the opportunity to work with him over the last 25 years. Chris retired two years ago, but fortunately, is still available for advice, and we always value his insights. This work was supported by NIH grants NS099333, NS072360, and NS104782 (to LJP and YHF) and by the William Bowes Neurogenetics Fund (to LJP and YHF).

Address correspondence to: Ying-Hui $\mathrm{Fu}$ 548B Rock Hall, MC-2922, 1550 4th Street, San Francisco, California 94143, USA. Phone: 415.502.2948; Email: Ying-Hui. Fu@ucsf.edu. Or to: Louis Ptáček, 548F Rock Hall, MC-2922, 1550 4th Street, San Francisco, California 94143, USA. Phone: 415.514.9305; Email:1jp@ucsf.edu.

1. Fu YH, et al. Variation of the CGG repeat at the fragile $\mathrm{X}$ site results in genetic instability: resolution of the Sherman paradox. Cell. 1991;67(6):1047-1058.

2. Ptácek LJ, et al. Identification of a mutation in the gene causing hyperkalemic periodic paralysis. Cell. 1991;67(5):1021-1027.

3. Fu YH, et al. An unstable triplet repeat in a gene related to myotonic muscular dystrophy. Science. 1992;255(5049):1256-1258.

4. Ptácek LJ, et al. Dihydropyridine receptor mutations cause hypokalemic periodic paralysis. Cell. 1994;77(6):863-868.

5. Plaster NM, et al. Mutations in Kir2.1 cause the developmental and episodic electrical phenotypes of Andersen's syndrome. Cell. 2001;105(4):511-519.

6. Botstein D, et al. Construction of a genetic linkage map in man using restriction fragment length polymorphisms. Am J Hum Genet. 1980;32(3):314-331.

7. Jones CR, et al. Familial advanced sleep-phase syndrome: A short-period circadian rhythm vari- ant in humans. Nat Med.1999;5(9):1062-1065.

8. Toh KL, et al. An hPer2 phosphorylation site mutation in familial advanced sleep phase syndrome. Science. 2001;291(5506):1040-1043.

9. Curtis BJ, et al. Extreme morning chronotypes are often familial and not exceedingly rare: the estimated prevalence of advanced sleep phase, familial advanced sleep phase, and advanced sleep-wake phase disorder in a sleep clinic population. Sleep. 2019;42(10):zsz148.

10. Konopka RJ, Benzer S. Clock mutants of Drosophila melanogaster. Proc Natl Acad Sci U S A. 1971;68(9):2112-2116.

11. Xu Y, et al. Functional consequences of a CKIdelta mutation causing familial advanced sleep phase syndrome. Nature. 2005;434(7033):640-644.

12. Xu Y, et al. Modeling of a human circadian mutation yields insights into clock regulation by PER2. Cell. 2007;128(1):59-70.

13. Zhang L, et al. A PERIOD3 variant causes a circadian phenotype and is associated with a seasonal mood trait. Proc Natl Acad Sci U S A. 2016;113(11):E1536-E1544.

14. Hirano A, et al. A novel human Cryptochrome 2 variant yields familial advanced sleep phase. eLife. 2016;5:e16695.

15. Kurien P, et al. TIMELESS mutation alters phase responsiveness and causes advanced sleep phase. Proc Natl Acad Sci US A. 2019;116(24):12045-12053.

16. He Y, et al. The transcriptional repressor DEC2 regulates sleep length in mammals. Science. 2009;325(5942):866-870.

17. Shi $\mathrm{G}$, et al. A rare mutation of $\beta_{1}$-adrenergic receptor affects sleep/wake behaviors. Neuron. 2019;103(6):1044-1055.e7.

18. Xing L, et al. Mutant neuropeptide S receptor reduces sleep duration with preserved memory consolidation. Sci Transl Med. 2019;11(514):eaax2014.

19. Shi G, et al. Mutations in metabotropic glutamate receptor 1 contribute to natural short sleep trait. Curr Biol. 2021;31(1):P13-P24.e4.

20. Ashbrook L, et al. Genetics of the human circadian clock and sleep homeostat. Neuropsychopharmacology. 2020;45(1):45-54 